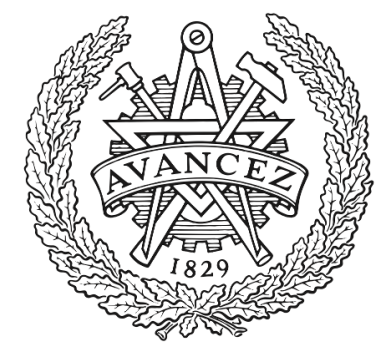

CHALMERS

UNIVERSITY OF TECHNOLOGY

\title{
Performance monitoring for live systems with soft FEC
}

Downloaded from: https://research.chalmers.se, 2023-04-26 13:23 UTC

Citation for the original published paper (version of record):

Yoshida, T., Mazur, M., Schröder, J. et al (2019). Performance monitoring for live systems with soft FEC. IET Conference Publications. http://dx.doi.org/10.1049/cp.2019.0958

N.B. When citing this work, cite the original published paper. 


\title{
PERFORMANCE MONITORING FOR LIVE SYSTEMS WITH SOFT FEC \\ Tsuyoshi Yoshida, ${ }^{1 *}$, Mikael Mazur ${ }^{3}$, Jochen Schröder ${ }^{3}$, Magnus Karlsson ${ }^{3}$ and Erik Agrell ${ }^{3}$
}

\author{
${ }^{1}$ Information Technology R\&D Centre, Mitsubishi Electric Corporation, Kamakura, Japan \\ ${ }^{2}$ Graduate School of Engineering, Osaka University, Suita, Japan \\ ${ }^{3}$ Fibre-Optic Communications Research Centre, Chalmers University of Technology, Gothenburg, Sweden \\ *Yoshida.Tsuyoshi@ah.MitsubishiElectric.co.jp
}

Keywords: Digital modulation, optical fibre communication, modulation coding, mutual information.

\begin{abstract} information from a histogram of L-values.

1 Introduction

System margin measurement is essential for network management. As a research or system installation test we can use known test bits, e.g. pseudo-random binary sequences, and compute various performance metrics with this knowledge. On the other hand, once the system has started carrying live traffic, the true transmitted bits would be unknown. Thus performance monitoring methods must be blind, i.e. without the knowledge of the true transmitted bits [1].
\end{abstract}

A technique to estimate the quality of live traffic transmission, without knowledge of the transmitted bits, is proposed. The technique, which is applicable to systems employing soft forward error correction coding, estimates the so-called asymmetric

For legacy systems using on-off keying without forward error correction (FEC), the bit error rate (BER) and corresponding Q-factor estimation were performed via a Gaussian approximation of the probability density functions (pdfs) [2]. Even with unknown data, means and standard deviations [2, Eq. (1)] of a binary signal can be derived from hard decisions. However, the BER must be sufficiently low and the decision reliability high, for the BER to be reliably estimated in such systems. After the emergence of hard-decision FEC, the BER before FEC decoding, pre-FEC BER (or Q), has been used for margin measurement relative to a threshold value that guarantees quasi-error-free performance after FEC decoding. The pre-FEC BER is then simply estimated from the ratio of flipped to total bits in FEC decoder.

For soft-decision FEC thresholds, information-theoretic performance metrics such as mutual information (MI) and generalised mutual information (GMI) are more reliable than pre-FEC BER for post-FEC performance prediction $[3,4]$. However, applying the GMI or other relevant information theoretic metrics [5-7] for live system performance monitoring is not straightforward, in the absence of blind estimation techniques for such metrics. Near the FEC threshold, pdf overlapping occurs much more frequently now than before, due to worse acceptable pre-FEC performance.

In this paper we will provide an estimation of the asymmetric information (ASI) [6], useful for systems running unknown live data, under the use of soft-decision FEC and reverse concatenation probabilistic shaping (PS) [8]. The ASI works as a soft FEC threshold [6,9], and the estimation error in ASI are shown to be $1.7 \%$ for the Gaussian channel and $3.5 \%$ for fibre-optic channels. These errors correspond to $0.3 \mathrm{~dB}$ and 0.6 $\mathrm{dB}$ signal-to-noise ratio ( $\mathrm{SNR}$ ) in the binary modulation case.

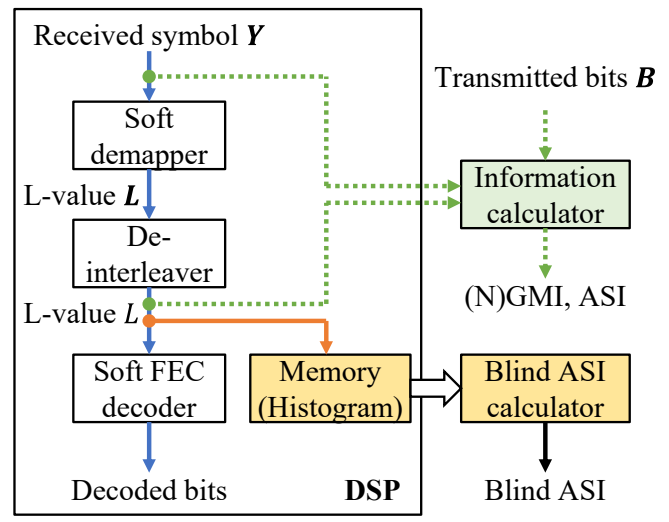

Fig. 1 Functions for blind performance monitoring in DSP.

\section{Preliminary and principle}

Fig. 1 shows our proposed blind performance monitoring functions for live systems. In this work we assume the use of a bitwise receiver. The received complex symbol $\boldsymbol{Y} \in \mathcal{R}^{2}$ is softly demapped to a posteriori L-values $\boldsymbol{L} \in \mathcal{R}^{m}$, deinterleaved to $L \in \mathcal{R}$, and decoded by a binary soft FEC decoder, where $\mathcal{R}$ and $m$ denote the real number set and number of bits per modulated two-dimensional symbol, resp. When carrying live traffic, the transmitted bits $\boldsymbol{B} \in \mathcal{B}^{m}$ are unknown, where $\mathcal{B}=\{0,1\}$. In this work we estimate the ASI without the knowledge of $\boldsymbol{B}$. We just need to add a function for monitoring an L-value histogram in real-time digital signal processing (DSP). Note that using the received symbol $\boldsymbol{Y}$ instead of $\boldsymbol{L}$ would also be possible, but then any approximation in soft demapping cannot be taken into account.

As FEC threshold for reverse concatenation PS, the normalised generalised mutual information (NGMI) [5], ASI [6] or 
achievable binary code rate [7] is useful. Recently NGMI and ASI were compared and showed different prediction performance [9]. Actually, the definition of NGMI and ASI are a bit different as

$$
\begin{gathered}
\text { NGMI }=1-\frac{H(\boldsymbol{B})-\max _{S>0} I_{S}^{\mathrm{gmi}}(\boldsymbol{B} ; \boldsymbol{Y})}{m} \\
\mathrm{ASI}=1-h\left(L_{\mathrm{a}}|| L_{\mathrm{a}} \mid\right)=1-\frac{H(\boldsymbol{B})-I_{S=1}^{\mathrm{gmi}}(\boldsymbol{B} ; \boldsymbol{Y})}{m} \\
I_{S}^{\mathrm{gmi}}(\boldsymbol{B} ; \boldsymbol{Y})=\mathbb{E}_{\boldsymbol{B}, \boldsymbol{Y}}\left[\log _{2} \frac{\mathrm{q}(\boldsymbol{b}, \boldsymbol{y})^{s}}{\sum_{\boldsymbol{b}} P_{\boldsymbol{B}}(\boldsymbol{b}) \mathrm{q}(\boldsymbol{b}, \boldsymbol{y})^{s}}\right]
\end{gathered}
$$

where $h, \mathbb{E}, \mathbb{q}(\boldsymbol{b}, \boldsymbol{Y})$ denotes differential entropy, expectation and auxiliary channel (a channel assumed in soft demapping) $[4,8,10]$, resp. Hence ASI $=$ NGMI in matched decoding $(s=$ $1)$ and ASI < NGMI in mismatched decoding cases. We here focus on ASI, which is based on symmetrised a posteriori Lvalue, or asymmetric $\mathrm{L}$-value, $L_{\mathrm{a}}=(-1)^{B} L$, where $L$ is the $\mathrm{L}$ value that will be fed into the FEC decoder, and $B$ is the corresponding transmitted bit [6]. As a possible example in practical implementation, the SNR of the auxiliary channel is set to a fixed FEC threshold value [11]. Then the SNRs of the received symbols carrying live traffic and that of the auxiliary channel will have a gap, e.g. several dB of system margin.
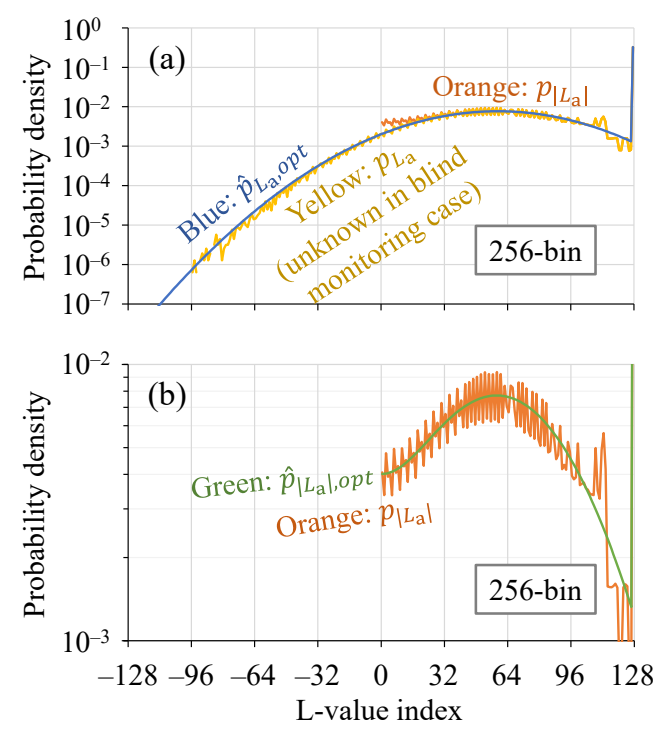

Fig. 2 Blind performance monitoring for PS-64-QAM having $H(\boldsymbol{B})$ of $4.1 \mathrm{bpcu}$. The pdf as a function of (a) symmetrised $a$ posteriori L-value $L_{\mathrm{a}}$ or (b) absolute value $\left|L_{\mathrm{a}}\right|$.

Figure 2 shows an example of L-values and the blind ASI estimation procedure for PS 64-ary quadrature amplitude modulation (QAM) at an SNR of $10 \mathrm{~dB}$. In the following simulation and experiment, we used more than $10^{6}$ samples of bitwise L-values. Constant composition distribution matching (CCDM) [12] is applied with the output block length of 1024 pulse amplitude modulation symbols. The QAM symbol entropy $H(\boldsymbol{B})$ is $4.1 \mathrm{bit} / \mathrm{channel}$ use (bpcu). We estimate the ASI from the pdf $p_{\left|L_{a}\right|}$ (orange in Fig. 2), which is obtained just after soft demapping. The operation principle consists of the following steps.

1. Prepare $K$ predetermined Gaussian distributions $\hat{p}_{L_{a}, k}^{\text {ini }}$ for $k=1, \cdots, K$, characterised by different means and variances, to be used as pdf candidates for $L_{a}$.
2. Set $\rho=p_{\left|L_{\mathrm{a}}\right|}(\max )$, where $L_{\mathrm{a}}$ is approximated by its quantised value and $\max$ and $\min$ denote the maximum and minimum quantised $L_{\mathrm{a}}$, resp.

3. For $k=1, \cdots, K$, let $\hat{p}_{L_{a}, k}(i)=(1-\rho) / \sum_{j=\min }^{\max -1} \hat{p}_{L_{a}, k}^{\text {ini }}(j)$ for $i=\min , \cdots, \max -1$ and $\hat{p}_{L_{\mathrm{a}}, k}(\max )=\rho$, to satisfy $\sum_{i=\min }^{\max } \hat{p}_{L_{\mathrm{a}}, k}(i)=1$.

4. Find the best $k=o p t$ from all $k \mathrm{~s}$ to minimise the mean square error between $p_{\left|L_{\mathrm{a}}\right|}$ (orange) and the $\hat{p}_{\left|L_{\mathrm{a}}\right|, k}$ (green), which is derived from $\hat{p}_{L_{a}, k}$. While we implement this as a full search over the predetermined distributions, other optimisation methods, e.g. gradient descent over the distribution parameters, can be employed.

5. Calculate the ASI, called blind ASI in this paper, by applying $\hat{p}_{L_{\mathrm{a}}, o p t}$ (blue) to $p_{L_{\mathrm{a}}}$ in (2).

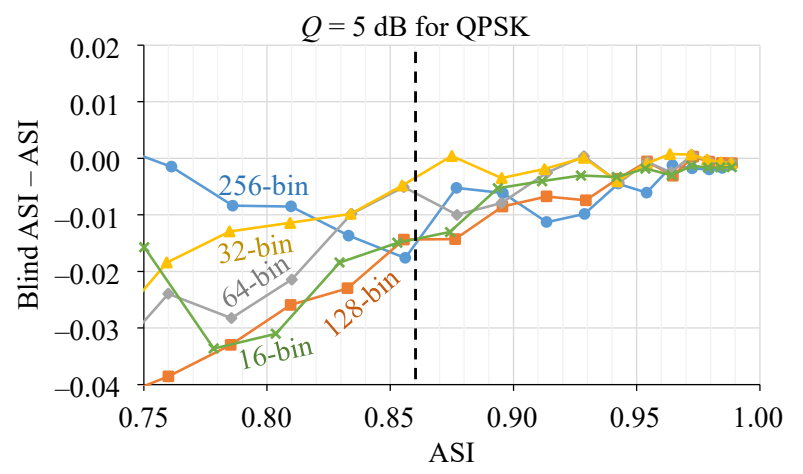

Fig. 3 Simulated absolute error in ASI estimation for PS-64QAM with $H(B)$ of 4.1 bpcu over the Gaussian channel.

Once the histogram $p_{\left|L_{\mathrm{a}}\right|}$ is stored in DSP, the above processing is implementable in an external software or field programmable gate array. For the case shown in Fig. 2, the ASI from the sampled $p_{\left|L_{\mathrm{a}}\right|}$ with knowledge of transmitted bits is 0.877 and that from the proposed estimation is 0.872 , so the absolute error is only 0.005 . Figure 3 shows the absolute estimation error by blind processing as a function of ASI when we swept the SNR from $5 \mathrm{~dB}$ to $15 \mathrm{~dB}$. The SNR in the auxiliary channel was set to a typical FEC threshold condition of ASI $=0.86$ [13], and the L-value was quantised to 4 to 8 bits, i.e. 16 to 256 bins. The absolute error in estimation is almost within 0.015 (the relative error is at most $1.7 \%$ ) at ASIs larger than 0.86 , which is the regime of practically interest. An ASI of 0.86 corresponds to a Q-factor (from BER) of $5 \mathrm{~dB}$ for binary modulation such as quaternary phase-shift keying (QPSK). The Q-factor can be also estimated from the chosen Gaussian distribution's mean and variance, i.e. $Q=$ mean $/ \sqrt{\text { variance. }}$.

In the computation of $h\left(L_{\mathrm{a}}|| L_{\mathrm{a}} \mid\right)$ in (2), step 5, $p_{\left|L_{\mathrm{a}}\right|}$ can be partially used instead of $\hat{p}_{\left|L_{\mathrm{a}}\right| \text {,opt }}$ to estimate ASI, but no significant difference was found. Note that once we have the ASI, the achievable information rate (AIR) can be estimated as $\mathrm{AIR}=H(\boldsymbol{B})-(1-\mathrm{ASI}) m$. 


\section{Experiments}

We experimentally examined the proposed method using the setup in Fig. 4, which was almost the same as the one used in [14]. A 51-tone optical comb was generated by an electrooptics comb generator with seeded light from an external cavity laser (ECL). Then the comb lines were separated into even/odd tones and modulated in an in-phase/quadrature (IQ) modulator. An arbitrary waveform generator created a 24 Gsymbol/s electrical signal, which was fed to the modulators. Polarisation-division multiplexing was emulated and neighbouring tones were decorrelated using optical interleavers (OIs). The generated comb-based $25 \mathrm{GHz}$ spaced 51-tone modulated signal was propagated through a recirculating loop consisting of $80 \mathrm{~km}$ standard single-mode fibre spans, erbium-doped fibre amplifiers, an optical bandpass filter for suppression of out-of-band amplified spontaneous emission noise, a wavelength-selective switch (WSS) for gain-tilt compensation and an acoustic optical modulator (AOM). There was no performance difference when including a polarisation scrambler inside the loop, so we removed it for loss reduction in the loop. The centre channel having a wavelength of $1545.32 \mathrm{~nm}$ was extracted by an optical filter, mixed with local oscillator light from an ECL, coherently detected and sampled in a digital sampling oscilloscope. The signal was recovered by pilot-based offline DSP [14]. The recovered constellations are shown as an inset in Fig. 4.

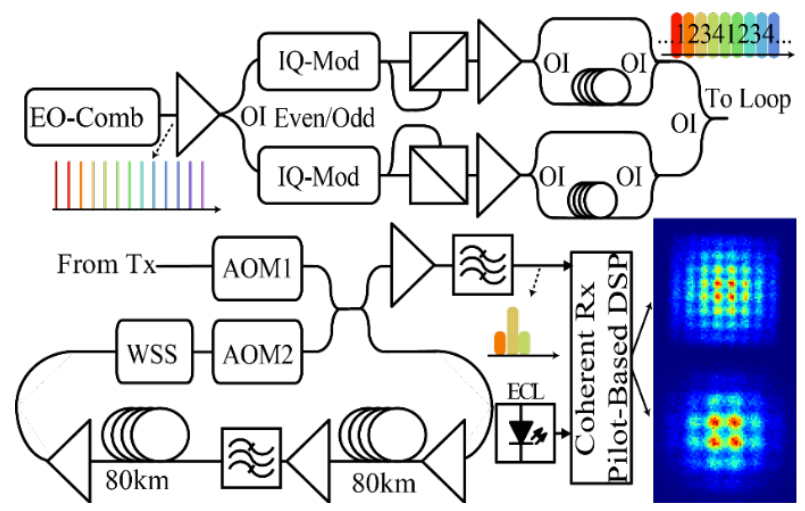

Fig. 4 Experimental setup. Insets are constellations for PS-64QAMs (5.7 bpcu at 5 loops and 4.6 bpcu at 13 loops, resp).

The examined modulation/shaping formats were 16-QAM, 64QAM, PS-16-QAM, PS-64-QAM and PS-256-QAM. The PS64QAM format was generated with CCDM [12], whose $H(\boldsymbol{B})$ were 4.1, 4.6, 5.2 and $5.7 \mathrm{bpcu}$, and the PS-16-QAM and PS256-QAM were generated with the DM in [15], whose $H(\boldsymbol{B})$ were 3.4 and $6.3 \mathrm{bpcu}$. The SNR assumed in the soft-demapper was set to a pre-set value per modulation/shaping case, assuming a typical FEC threshold of ASI $=0.86$ [13] regardless of the number of loops. The number of L-value bins were set to 32 bins as a possible value in a deployable DSP. Figure 5 shows the ASI with transmitted bit knowledge (solid lines) and by the proposed method without such knowledge (dashed lines). The markers for base constellations 16, 64 and 256-QAMs are coloured in green/red, blue/orange and yellow/purple. In the regime where the ASI is more than 0.86 (black line), the maximum absolute error is within 0.03 , so the maximum estimation error is $3.5 \%$. A potential cause of this two times larger estimation error for the fibre-optic channel than the Gaussian channel may be that analogue signal distortion makes the L-value distribution more non-Gaussian, which is also influenced by the normalisation and quantisation of L-values, and the finite number of Gaussian distributions tested (here $K=8192$ ). Below the assumed FEC threshold ASI of 0.86 , the prediction performance becomes worse due to the pre-set fixed soft-demapping. The prediction performance was not improved even when the ASIs of each bit-level were estimated separately and then averaged. We have also monitored the Q-factor, and then the absolute error was less than $1 \mathrm{~dB}$ at a true Q-factor from 5 to $10 \mathrm{~dB}$.

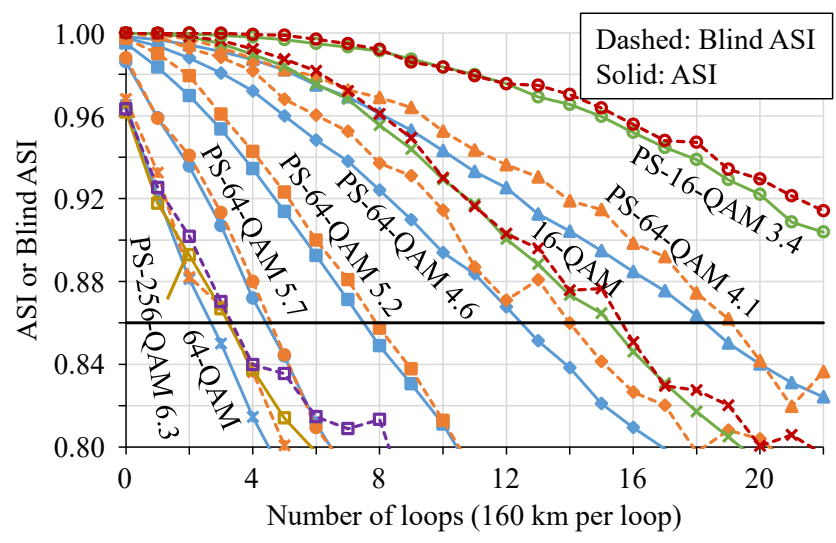

Fig. 5 Experimental results.

\section{Conclusion}

Soft-decision FEC thresholds are efficiently estimated from bitwise L-value histogram using a Gaussian approximation. Experiments show typical estimation errors within 3.5\%, which corresponds to an SNR of around $0.6 \mathrm{~dB}$ in the regime of ASI $=0.85-0.95$ in the case of a binary modulation. This accuracy is comparable with typical SNR estimation methods. The proposed method needs minimal overhead in hardware, i.e. just a memory for saving the L-value histogram, so it is feasible for live system performance monitoring.

\section{Acknowledgements}

This work was partly supported by "The research and development of innovative optical network technology as a new social infrastructure" of the Ministry of Internal Affairs and Communications, and "Massively Parallel and Sliced Optical Network," the Commissioned Research of National Institute of Information and Communications Technology (NICT), Japan, and partly by the Swedish Research Council, VR (grant 2014-06138). We thank Assoc. Prof. Koji Igarashi of Osaka University for fruitful discussions.

\section{References}

[1] Dong, Z., Khan, F. N., Sui, Q., et al.: 'Optical performance monitoring: a review of current and future technologies', J. Lightw. Technol., 2016, 34, (2), pp. 525-543 
[2] Bergano, N. S., Kerfoot, F. W., Davidson, C. R.: 'Margin measurement in optical amplifier systems', Photon. Technol. Lett., 1993, 5, (3), pp. 304-306

[3] Schmalen, L., Alvarado, A., Rios-Müller, R.:

'Performance prediction of nonbinary forward error correction in optical transmission experiments', J. Lightw.

Technol., 2017, 35, (4), pp. 1015-1027.

[4] Alvarado, A., Agrell, E., Lavery, D., et al.: 'Replacing the soft FEC limit paradigm in the design of optical communication systems', J. Lightw. Technol., 2016, 34, (2), pp. 707-721

[5] Cho, J., Schmalen, L., Winzer, P. J.: 'Normalized generalized mutual information as a forward error correction threshold for probabilistically shaped QAM'. Proc. Eur. Conf. Opt. Commun. (ECOC), Sweden, Sep. 2017, p. M.2.D.2

[6] Yoshida, T., Karlsson, M., Agrell, E.: 'Performance metrics for systems with soft-decision FEC and probabilistic shaping', Photon. Technol. Lett., 2017, 29, (23), pp. 21112114

[7] Böcherer, G.: 'Achievable rates for probabilistic shaping', http://www.arxiv.org/pdf/1707.01134v5

[8] Böcherer, G., Steiner, F., Schulte, P.: 'Bandwidth efficient and rate-matched low-density parity-check coded modulation', IEEE Trans. Commun., 2015, 33, (20), pp. $4338-4352$

[9] Zhang, S., Yaman, F., Mateo, E., et al.: 'On the performance metric and design of non-uniformly shaped constellation'. Proc. Opt. Fib. Commun. Conf. (OFC), USA, March 2019, p. W1D.7

[10] Buchali, F., Steiner, F., Böcherer, G., et al.: 'Rate adaptation and reach increase by probabilistically shaped 64 QAM: an experimental demonstration', J. Lightw. Technol., 2016, 34, (7), pp. 1599-1609

[11] Yoshida, T, Matsuda, K., Kojima, K, et al.: 'Hardwareefficient precise and flexible soft-demapping for multidimensional complementary APSK signals', Proc. Eur. Conf. Opt. Commun. (ECOC), Germany, Sep. 2016, p.

\section{Th.2.P2.SC3.27}

[12] Schulte, P., Böcherer, G.: 'Constant composition distribution matching', IEEE Trans. Inf. Theory, 2016, 62, (1), pp. 430-434

[13] Sugihara, K., Miyata, Y., Sugihara, T.: 'A spatiallycoupled type LDPC code with an NCG of $12 \mathrm{~dB}$ for optical transmission beyond $100 \mathrm{~Gb} / \mathrm{s}$. Proc. Opt. Fib. Commun. Conf. (OFC), USA, March 2013, p. OM2B.4

[14] Mazur, M., Schröder, J., Lorences-Riesgo, A., et al.: 'Optimization of low-complexity pilot-based DSP for high spectral efficiency 51×24Gbaud PM-64QAM transmission'. Proc. Eur. Conf. Opt. Commun. (ECOC), Italy, Sep. 2018, p. Mo4F.2

[15] Yoshida, T., Karlsson, M., Agrell, E.: 'Low-complexity variable-length output distribution matching with periodical distribution uniformalization'. Proc. Opt. Fib. Commun. Conf. (OFC), USA, March 2018, p. M4E.2 